\title{
Décadrages Décadrages
}

cinéma, à travers champs Cinéma, à travers champs

11 | 2007

Terrence Malick

\section{Editorial}

Alain Boillat

\section{OpenEdition}

Journals

Édition électronique

URL : https://journals.openedition.org/decadrages/391

DOI : 10.4000/decadrages.391

ISSN : 2297-5977

\section{Éditeur}

Association Décadrages

\section{Édition imprimée}

Date de publication : 1 octobre 2007

Pagination : 4-7

ISBN : 978-2-9700582-6-7

ISSN : 2235-7823

Référence électronique

Alain Boillat, «Editorial », Décadrages [En ligne], 11 | 2007, mis en ligne le 01 octobre 2007, consulté le 05 avril 2022. URL : http://journals.openedition.org/decadrages/391 ; DOI : https://doi.org/10.4000/ decadrages.391

(B Décadrages 


\section{Editorial}

par Alain Boillat

1 Filmographie de Terrence Malick:

- Lanton Mills (court métrage, 1969)

- Badlands (La Balade sauvage, 1973). Avec Martin Sheen, Sissy Spacek, Warren Oates,...

- Days of Heaven (Les Moissons du ciel, 1978). Avec Richard Gere, Brooke Adams, Sam Shepard, Linda Manz,...

- The Thin Red Line (La Ligne rouge, 1998). Avec Adrien Brody, James Caviezel, Nick Nolte, John Cusack, Sean Penn, Elias Koteas,...

- The New World (Le Nouveau Monde, 2005). Avec Colin Farrell, Q'Orianka Kilcher, Christian Bale, Christopher Plummer,...

2 La Ligne rouge, Editions de la Transparence, Chatou, 2005.
Dans le paysage de la production hollywoodienne, Terrence Malick est une figure singulière. Avec seulement quatre longs métrages réalisés sur une période de plus de trente ans $\mathbf{1}$, il pourrait passer pour un "génie méticuleux» à la Kubrick si l'on pouvait plus aisément asseoir son statut d'auteur et si ses deux derniers films ne comportaient pas une démesure qui en fait des œuvres "problématiques» (au sens positif du terme). En dépit de sa réserve (il ne se livre qu'exceptionnellement à l'exercice de l'entretien) et du caractère épisodique de ses réalisations, Malick a acquis la reconnaissance de la critique et le respect du milieu hollywoodien. En témoigne le fait qu'en 1997, ce cinéaste qui n'a plus tourné depuis vingt ans se voit confier un film, The Thin Red Line (La Ligne rouge, 1998), dont le budget dépasse les cinquante millions de dollars et dans lequel se côtoient plusieurs stars masculines du moment (Sean Penn, Adrien Brody, Jim Caviezel, Nick Nolte), certains acteurs comme George Clooney ou John Travolta acceptant même de n'y faire qu'une brève apparition. Si l'activité de scénariste a permis à Malick de ne jamais s'éloigner d'Hollywood, le parcours de cet intellectuel venu au cinéma après avoir étudié la philosophie et traduit Heidegger demeure atypique.

A la discrétion du cinéaste (qui n'apparaît par exemple qu'en creux dans le making of de son dernier film) correspond l'indiscutable carence de la littérature portant sur son travail: aucune monographie ne lui a été consacrée, et il n'y a à ce jour dans le domaine francophone guère que l'opuscule de Michel Chion qui porte sur l'un de ses films ${ }^{2}$. La richesse de The New World (Le Nouveau Monde, 2005) sorti dernièrement nous a incités à consacrer à ce réalisateur le dossier de ce numéro, parution qui sera accompagnée dès le 6 février 2008 d'une rétrospective à la Cinémathèque suisse comprenant, en plus des quatre longs métrages de Malick, le film Pocket Money (Stuart Rosenberg, 1972) qu'il a coscénarisé. Nous espérons ainsi ouvrir la voie à de plus amples réflexions sur cette œuvre protéiforme, tiraillée entre grandiloquence et sobriété, entre l'esthétisation de l'image et le tourbillon d'un montage tantôt haché, tantôt d'une fluidité époustouflante. Le Nouveau Monde constitue peut-être sur le plan stylistique le film le plus abouti de Malick en termes de conciliation de la saccade et du flux, de la distance et de l'immersion, du regard 
critique et de l'adhésion totale - avec tout ce que ce va-et-vient peut comporter d'ambigu sur le plan «idéologique».

$\mathrm{Au}$ vu des caractéristiques esthétiques des films de Malick, on ne s'étonnera pas de trouver dans plusieurs contributions de ce dossier une attention portée à des paramètres de type "rythmique» convoqués de façon particulièrement manifeste dans les deux dernières réalisations du cinéaste. Examinant la question de la musicalité et la façon dont le Prélude de L'Or $d u$ Rhin imprègne à différents niveaux l'esthétique et les significations du Nouveau Monde, Laurent Guido inscrit le travail du cinéaste dans une recherche sur le rythme qu'il corrèle à une tradition de représentation profondément ancrée dans l'histoire américaine ; dans l'article "Entre distance et immersion: des voix qui glissent sur le monde", un constat similaire est proposé en ce qui concerne l'usage des voix-over, procédé dont Malick use dans tous ses films en lui conférant un rôle qui excède très largement celui, traditionnel, qui consiste à transmettre des informations narratives. La dimension sonore est également analysée au niveau des bruits par Martin Barnier, qui souligne combien la bande-son de La Ligne Rouge contribue de manière quasi imperceptible mais néanmoins décisive à construire les espaces qu'habitent les personnages (mais aussi ceux dont ils sont habités) et à poser une atmosphère, cédant peu aux effets démonstratifs devenus caractéristiques des films de guerre depuis la généralisation du multipiste.

Jouant avec les silences, les nappes de musique symphonique et le surgissement énigmatique des voix, introduisant constamment des phases de suspension dominées par l'itératif, Malick nous emporte dans le mouvement des cours d'eau qu'empruntent soldats et pionniers. Son cinéma est à la fois bavard puisque s'y enchevêtrent constamment des voix-over (quoiqu'il s'agisse, justement, plus de voix et de "grains» que véritablement de paroles) et proche du cinéma muet en raison d'un montage périodiquement affranchi de l'ancrage spatio-temporel induit par la voix synchrone. Dès Badlands (La Balade sauvage, 1973) ${ }^{3}$, ainsi que le montre Frank Steiger, la mise en scène des prises de parole provoque une désolidarisation d'avec les personnages, qui demeurent étrangers les uns aux autres et à eux-mêmes, aliénés par l'appropriation involontaire de discours stéréotypés. Au vu du traitement singulier accordé par Malick à une parole poussée aux limites de son inefficacité (règne de l'infraverbal, vanité du discours humain face à la domination impassible de la nature), on pourrait avancer qu'il y a chez lui quelque chose du cinéma de la fin des années 1920, de cet idéal du film «sonore» (en opposition au "parlant») qu'ont momentanément nourri, à l'époque de la généralisation des talkies, certains théoriciens marqués par le modèle
3 Selon Michel Chion (op. cit., p. 99, note 3), le terme "balade" s'est vu pourvu d'un second "I" à l'occasion de la sortie vidéo du film. Nous nous en tiendrons à l'orthographe originelle. 
4 Actualisant cette comparaison, le récent western The Assassination of Jesse James by the Coward Robert Ford (Andrew Dominik, 2007) emprunte significativement de nombreux traits stylistiques aux deux premiers films de Malick.

5 Rappelons que le premier long métrage de Malick constitue encore, une trentaine d'années après sa sortie, un modèle pour un cinéaste helvétique comme Christophe Marzal. Voir Décadrages n 4-5, printemps 2005, p. 109 (analyse de Au large de Bad Ragaz) et p. 123 (entretien avec le cinéaste). musicaliste. Les chants de La Ligne rouge ne sont pas sans animer l'environnement comme ceux d'Hallelujah (King Vidor, 1929), et on pourrait trouver un ancêtre au Nouveau Monde du côté d'un film comme Ombres blanches (White Shadowes in the South Seas, 1928) qui, réalisé conjointement par W. S. Van Dyke et le documentariste Robert Flaherty, associe deux facettes que l'on retrouve chez Malick: d'une part un souci d'authenticité accompagné du respect des terres vierges et des populations indigènes, d'autre part une envolée dans une romance naïve et un lyrisme échevelé. Si la relation de l'homme à la nature est empruntée aux auteurs transcendantalistes tels que Thoreau, Whitman ou Emerson - tissu référentiel dont François Bovier commente la richesse tout en soulignant les limites de l'appropriation par Malick de cet intertexte -, l'intrigue amoureuse demeure associée à la récente popularisation proposée par Walt Disney (Pocahontas, Mike Gabriel et Eric Goldberg, 1995).

En effet, comme le remarque la plupart des intervenants de ce dossier, les films de Malick menacent constamment de basculer dans le cliché ou plutôt, pour inverser cette logique, procèdent véritablement d'un traitement des clichés qu'ils exhibent ou retravaillent. En ce sens, la référence à un genre cinématographique est avant tout le lieu d'insignes décalages: La Ligne rouge fait glisser le film de guerre du côté de la réflexion philosophique; Le Nouveau Monde échappe à toute classification générique - même si, comme le suggère François Bovier, c'est peutêtre en termes de renouvellement du western qu'il faudrait l'appréhender (comme tous ses films d'ailleurs) 4 ; Badlands, road movie sanglant de type Bonnie and Clyde (Arthur Penn, 1967) ${ }^{\mathbf{5}}$, ne s'inscrit pas tant dans l'effervescence du «New Hollywood» qu'il ne présente, au travers d'une parole féminine désinvolte et distanciée, un James Dean de pacotille dont la représentation suggère l'inanité des aspirations nostalgiques du moment. Quant à Days of Heaven (Les Moissons du ciel, 1978), Malick y étouffe la veine mélodramatique en déplaçant l'intérêt pour les personnages vers la nature qui les environne, ainsi que le montre Jean-Michel Durafour dans une optique "figurale» qui permet de prendre toute la mesure de l'exploitation par le cinéaste de la dimension purement sensorielle des images et des sons. Le chatoiement des rayons du soleil sur les champs de blé des Moissons $d u$ ciel ou les infimes variations de luminosité qui modèlent le rendu de la végétation luxuriante filmée dans La Ligne rouge ne sont pas (seulement) des afféteries d'esthète pompier: de telles «mues de la nature» (pour reprendre l'expression de Durafour) constituent l'objet même de ces films. Cette tendance à évincer les êtres pour retourner à la terre première est constante chez Malick, qui, à l'instar de Laughton dans The Night of the Hunter (La Nuit du chasseur, 1955), 
ne craint pas de ponctuer ses films d'inserts qui semblent issus de films animaliers. Malick ose l'hétérogénéité tout en se fixant le défi, souvent atteint, de l'harmonie.

La rubrique "cinéma suisse» est entièrement consacrée à l'histoire de la production nationale, abordée au travers d'objets filmiques de nature fort différente. Claire-Lise Debluë se penche sur deux films d'entreprise réalisés par l'OSEC (Office Suisse d'Expansion Commerciale) dont elle examine le contexte de production sur les plans économique et politique, articulant l'analyse des discours - elle démontre combien la volonté de rationalisation se situe au centre des réflexions de l'OSEC sur le cinéma - avec celle des films, ces derniers thématisant précisément au niveau formel des questions relatives à la taylorisation. Exploitant des sources rares (versions successives des scénarios, correspondance, etc.), Alexandra Walther étudie quant à elle la genèse de La Suisse s'interroge, ensemble de courts métrages réalisés par Henry Brandt à l'occasion de l'Expo 64. Dans une optique conciliant une analyse fonctionnelle de type narratologique et une attention portée tant aux dynamiques abstraites sous-tendant la trame filmique qu'à leurs figurations concrètes, Alain Freudiger revient sur l'une des œuvres célèbres du Nouveau cinéma suisse, Les Arpenteurs (1972) de Michel Soutter, pour en dégager certains motifs récurrents et discuter les principes régissant leur déploiement. En filant les manifestations multiples du concept de "mesure", Freudiger met en lumière une géométrie interne aux Arpenteurs dont il montre qu'elle est fondée, selon une logique quasi burlesque, sur les mouvements rythmés des personnages, les déplacements des corps, l'organisation des dialogues et la circulation des objets. A l'issue de ces trois contributions qui offrent un regard inédit sur des réalisations helvétiques, Pierre-Emmanuel Jaques rend compte d'une publication récente, l'Histoire du cinéma suisse, 1966-2000. Dirigé par Maria Tortajada et Hervé Dumont, cet ouvrage somme est le résultat d'un travail collectif auquel plusieurs collaborateurs réguliers de Décadrages ont pris part. Il ne reste qu'à espérer que la richesse des informations contenues dans ces deux volumes saura susciter des recherches dans un champ d'étude auquel la rubrique régulière de notre revue entend fournir une modeste contribution. 